\title{
On the Possibility to Create a Compatible-Complete Unary Comparison Method for Evolutionary Multiobjective Algorithms
}

\author{
Giovanni Lizárraga \\ Lizárraga \\ Center of Research in \\ Mathematics \\ Jalisco S/N, Valenciana \\ Guanajuato, México \\ giovanni@cimat.mx
}

\author{
Arturo Hernández \\ Aguirre \\ Center of Research in \\ Mathematics \\ Jalisco S/N, Valenciana \\ Guanajuato, México \\ artha@cimat.mx
}

\author{
Salvador Botello Rionda \\ Center of Research in \\ Mathematics \\ Jalisco S/N, Valenciana \\ Guanajuato, México \\ botello@cimat.mx
}

\begin{abstract}
There are several studies on the desirable properties that a performance measure for evolutionary multiobjective algorithms must have. One of these properties is called "compatibility and completeness". There is a theorem that proves that in the general case, it is not possible to create a unary comparison method with the property mentioned before. Many important conclusions have been derived from this theorem, so its correctness is fundamental for future research. In this work we demonstrate that under practical conditions, the theorem mentioned before does not hold.
\end{abstract}

\section{Categories and Subject Descriptors}

I.2.m [Artificial Inteligence]: Miscellaneous

\section{General Terms}

Measurement

\section{Keywords}

Multiobjective optimization, performance measures, Pareto optimality

\section{DISCUSSION}

Multiobjective optimization using Evolutionary Computation $(M O E C)$ is an area of research that has grow a lot in recent years. For a survey in general terms of MOEC, like Pareto optimality, non-dominated set (NS), objective function, objective function space, see [1] [?].

An open problem in MOEC is the creation of performance measures $(P M)$ to evaluate the performance of different Multiobjective Evolutionary Algorithms (MOEA). The MOEAs generate non-dominated sets of finite size. So the evaluation of the MOEAs can be reduced to evaluate the NSs that they generate.

Unary performance measures $(U P M)$ are methods that evaluate how good is a non-dominated set and transform this evaluation in a real number. Let $\mathbf{I}$ be a finite vector of UPM and $\mathbf{I}(A)$ the vector of evaluations of the elements of $\mathbf{I}$

Copyright is held by the author/owner(s).

GECCO'08, July 12-16, 2008, Atlanta, Georgia, USA.

ACM 978-1-60558-130-9/08/07. for the NS $A$. Let $E(\mathbf{I}(A), \mathbf{I}(B))$ be an interpretation function that takes the vectors $\mathbf{I}(A)$ and $\mathbf{I}(B)$ for two NSs $A$ and $B$, and evaluate if $A$ is better than $B$. The combination $C_{\mathbf{I}, E}$ of $\mathbf{I}$ and $E$ is called a unary comparison method(UCM) [3] .

In [3], it was defined a property for performance measures known as "compatibility and completeness" $(C C)$. This is a very important property, because if a $\mathrm{PM}$ is compatible and complete than it is able to detect whether a NS is better than another. In that same work, the following theorem was demonstrated.

THEOREM 1. For multiobjective problems (MOP) with 2 or more objectives, there exits no unary comparison method with a finite number $k$ of unary performance measures, that is compatible and complete.

To prove this, it is assumed that there exists a unary comparison method that is compatible and complete ( $C C U C M)$, then it is shown that this assumption leads to a contradiction. The direct consequence is that unary comparison methods are limited in their capability to evaluate NSs. In order to prove Theorem 1, Ziztler et al [3] first proved the following Lemma:

LEMMA 1. If there exist a CCUCM with $\boldsymbol{I}=\left(I_{1}, I_{2}, \ldots, I_{k}\right)$, and an interpretation function $E$, then for all NSs $A$ and $B$ with $A \neq B$ there is at least one $I_{j}$ in $\boldsymbol{I}$ such that $I_{j}(A) \neq$ $I_{j}(B)$.

In other words for a CCUCM, if $A \neq B$ then $\mathbf{I}(A) \neq \mathbf{I}(B)$. We present a demonstration of Theorem 1 that is equivalent to the one presented in [3]. But first we introduce some concepts. We represent the cardinal number of countable sets with $a$, the cardinal number of the continuous line with $c$ and the cardinal number of the power set of a continuous line with $f$. It is proved that $a<c<f$ [2]. The cardinal number of a set $A$ is represented with $\phi(A)$.

Define $\Upsilon$ as the set of all different vectors I we can generate with the evaluations of $k$ performance measures. We have that $\phi(\Upsilon)=c$, because the set of different vectors we can generate with $k$ real numbers is equivalent to $R^{k}[2]$. And, the cardinality of $R^{k}$ is $c[2]$ so $\phi(\Upsilon)=c$.

Let $Z$ be the space of objective functions for a MOEA with $n$ objectives. We consider for the rest of the paper the most general case where $Z=R^{n}$. Define $\Omega$ as the set 
of all NSs we can generate from $Z$. The demonstration of Theorem 1 is the following.

Proof of Theorem 1. The demonstration is as follows:

(1) $\phi(\Omega)=f$. See [?].

(2) $\phi(\Upsilon)=c$.

(3) We need to make an injection from $\Omega$ to $\Upsilon$. Because of Lemma 1.

(4) It is impossible to make an injection from $\Omega$ to $\Upsilon$. Because of (1), (2) and because it is impossible to make an injection from a set of cardinality $f$ to a set of cardinality $c[2]$.

In step (4) of the demonstration of Theorem 1 we have a contradiction. From this, we conclude that a CCUCM cannot exist. We call this contradiction "the cardinality contradiction".

Unfortunately, there is a flaw in the demonstration of Theorem 1 (both in the one presented here and the one in [3]). The problem is that it is assumed implicitly that we are going to compare NSs of infinite size. In practice, all NSs to compare have finite cardinality because MOEAs can only generate NSs with a finite number of elements. When we consider this in the demonstration of Theorem 1, the cardinality contradiction vanishes. To prove this we first demonstrate the following lemma:

Lemma 2. Let $Z=R^{n}$. Define $\Omega_{<a}$ as the set of all NSs of finite size that we can generate from $Z$. The cardinal number of $\Omega_{<a}$ is $c$.

Proof. Define $\Omega_{k}$ as the set of all NSs of size $k$ that we can generate from $Z$. We can represent $\Omega_{<a}$ with the following sum:

$$
\Omega_{<a}=\Omega_{1}+\Omega_{2}+\Omega_{3}+\ldots
$$

Note that this sum have countable many elements, because we can make a bijection between the positive integers and the elements of the sum. For this we only need to associate each positive integer $k$ with the corresponding $\Omega_{k}$.

(1): $\phi\left(\Omega_{k}\right) \leq \phi\left(\mathbf{R}^{k n}\right)$. Because we can make an injection from $\Omega_{k}$ to $\mathbf{R}^{k n}$. In order to make this injection, for all nondominated sets $A \in \Omega_{k}$, sort the vectors in $A$ with respect to their components. With the ordered vectors we can make a different $k n$-tuple of real numbers for each NS $A$. These $k n-$ tuples are in the form $\left\langle v_{1}^{1}, v_{2}^{1}, \ldots, v_{n}^{1}, v_{1}^{2}, \ldots, v_{n}^{k}>\right.$ where $v_{i}^{j}$ represent the $i$-th component of the $j$-th ordered vector in $A$. Associate each $k n$-tuple with the corresponding point $\left(v_{1}^{1}, v_{2}^{1}, \ldots, v_{n}^{1}, v_{1}^{2}, \ldots, v_{n}^{k}\right)$ in $\mathbf{R}^{k n}$ to obtain an injection from $\Omega_{k}$ to $\mathbf{R}^{k n}$.

(2): $\phi\left(\Omega_{\leq a}\right) \leq \phi\left(\mathbf{R}^{n}+\mathbf{R}^{2 n}+\mathbf{R}^{3 n} \ldots\right)$. Because each $\Omega_{k}$ has a smaller or equal cardinality than the corresponding $\mathbf{R}^{k n}$. The different $\mathbf{R}^{k n}$ s are mutually disjoint sets, so the union of the $\mathbf{R}^{2 n}$ S has a greater or equal cardinality than the union of the $\Omega_{k}$ s.

(3): $\phi\left(\Omega_{\leq a}\right) \leq \phi\left(\mathbf{R}^{n}\right)+\phi\left(\mathbf{R}^{2 n}\right)+\phi\left(\mathbf{R}^{3 n}\right)+\ldots$ Because the cardinality of the union of mutually disjoint sets is equal to the sum of their cardinal numbers.

(4): $\phi\left(\Omega_{\leq a}\right) \leq c+c+\ldots$. Because the cardinal number of $\mathbf{R}^{k n}$ is $c$ [2].

(5): $\phi\left(\Omega_{\leq a}\right) \leq c$. Because the sum of countable many $c$ is equal to $c[2]$.
(6): $\phi\left(\Omega_{1}\right)=\phi(Z)$. Because we can make a bijection between $\Omega_{1}$ and $Z$. For this, associate each element $z \in Z$ with the corresponding $\{z\} \in \Omega_{1}$.

(7): $\phi\left(\Omega_{1}\right)=c$. Because $\phi(Z)=\phi\left(R^{n}\right)=c$.

(8): $\Omega_{1} \subset \Omega_{\leq a}$. Because of the definition of $\Omega_{1}$ and $\Omega_{\leq a}$.

(9): $\phi\left(\Omega_{1}\right) \leq \phi\left(\Omega_{\leq a}\right)$. Because the subsets of a set $A$ have a lower or equal cardinality than $A[2]$.

(10): $c \leq \phi\left(\Omega_{\leq a}\right)$. Because of (7) and (9).

(11): $c \leq \phi\left(\Omega_{\leq a}\right) \leq c$. Because of (5) and (10).

(12): $\phi\left(\Omega_{\leq a}\right)=c$. Because of (11).

Now, it is easy to prove the following theorem:

THEOREM 2. If we only compare non-dominated sets $A \in$ $\Omega_{<a}$, the cardinality contradiction vanishes.

Proof. The argumentation is the following.

(1) $\phi\left(\Omega_{\leq a}\right)=c$. Because of Lemma 2 .

(2) $\phi(\Upsilon)=c$.

(3) We need to make and injection from $\Omega_{\leq a}$ to $\Upsilon$. Because of Lemma 1.

(4) It is possible to make an injection from $\Omega_{\leq a}$ to $\Upsilon$. Because of (1), (2).

\section{CONCLUSIONS}

We proved that the demonstration of Theorem 1 does not hold in practical conditions (non-dominated sets of finite size). As a consequence we can not affirm anymore that it is impossible to construct a CCUCM. So as a future work we have two possibilities, to find another demonstration for Theorem 1 or to demonstrate that CCUCM are possible in practice.

\section{REFERENCES}

1] C. A. Coello, D. Van Veldhuizen, and G. B. Lamont. Evolutionary Algorithms for Solving Multi-Objective Problems. Kluwer Academic/Plenum Publishers, New York, USA, 2002.

[2] K. Deb. Multi-objective Optimization Using Evolutionary Algorithms. John Wiley and Sons, Chichester, UK, 2001.

[3] E. Kamke. Theory of Sets. Dover Publications, Inc., New York, USA, 1950.

[4] G. Lizarraga, A. Hernandez, and S. Botello. On the Possibility to Create a Compatible-Complete Unary Comparison Method for Evolutionary Multiobjective Algorithms. Technical report i-08-09 (cc) 15.04.2008, Centro de Investigacion en Matematicas, 2008.

[5] E. Zitzler, L. Thiele, M. Laumanns, C. M. Fonseca, and V. Grunert da Fonseca. Performance Assessment of Multiobjective Optimizers: An Analysis and Review. IEEE Transactions on Evolutionary Computation, 7(2):529-533, May 2003. 